ARTICLE

DOI: $10.1038 /$ s41467-018-06208-y

\title{
EVI1 overexpression reprograms hematopoiesis via upregulation of Spi1 transcription
}

\author{
Edward Ayoub (1) 1,2,3, Michael P. Wilson', Kathleen E. McGrath (1) 2,4, Allison J. Li2,5, Benjamin J. Frisch 2,5, \\ James Palis ${ }^{2,4}$, Laura M. Calvi (iD) ${ }^{2,5}$, Yi Zhang (1D) ${ }^{1,2} \&$ Archibald S. Perkins (1D ${ }^{1,2}$
}

$\operatorname{lnv}(3 q 26)$ and $t(3: 3)(q 21 ; q 26)$ are specific to poor-prognosis myeloid malignancies, and result in marked overexpression of EVI1, a zinc-finger transcription factor and myeloidspecific oncoprotein. Despite extensive study, the mechanism by which EVI1 contributes to myeloid malignancy remains unclear. Here we describe a new mouse model that mimics the transcriptional effects of $3 q 26$ rearrangement. We show that EVI1 overexpression causes global distortion of hematopoiesis, with suppression of erythropoiesis and lymphopoiesis, and marked premalignant expansion of myelopoiesis that eventually results in leukemic transformation. We show that myeloid skewing is dependent on DNA binding by EVI1, which upregulates SpiT, encoding master myeloid regulator PU.1. We show that EVI1 binds to the $-14 \mathrm{~kb}$ upstream regulatory element (-14kbURE) at SpiT; knockdown of Spi7 dampens the myeloid skewing. Furthermore, deletion of the -14kbURE at Spil abrogates the effects of EVI1 on hematopoietic stem cells. These findings support a novel mechanism of leukemogenesis through EVI1 overexpression.

\footnotetext{
${ }^{1}$ Department of Pathology and Laboratory Medicine, University of Rochester Medical Center, Rochester, NY 14642, USA. ${ }^{2}$ James P. Wilmot Cancer Institute, University of Rochester Medical Center, Rochester, NY 14642, USA. ${ }^{3}$ Department of Pharmacology and Physiology, University of Rochester Medical Center, Rochester, NY 14642, USA. ${ }^{4}$ Department of Pediatrics and Center for Pediatric Biomedical Research, University of Rochester Medical Center, Rochester, NY 14642, USA. ${ }^{5}$ Department of Medicine, University of Rochester Medical Center, Rochester, NY 14642, USA. These authors contributed equally: Edward Ayoub, Michael P. Wilson. These authors jointly supervised this work: Yi Zhang, Archibald S. Perkins. Correspondence and requests for materials should be addressed to Y.Z. (email: yi_zhang@urmc.rochester.edu) or to A.S.P. (email: archibald_perkins@urmc.rochester.edu)
} 
C hromosomal rearrangements at $3 \mathrm{q} 26$ are associated with poor-prognosis acute myeloid leukemia $(\mathrm{AML})^{1}$, myelodysplastic syndromes $(\mathrm{MDS})^{2}$, and myeloproliferative neoplasms $(\mathrm{MPN})^{3}$, and cause activation of MECOM, a gene that encodes multiple zinc-finger ( $\mathrm{ZF})$ transcription factor isoforms, including three isoforms of EVI1. These isoforms, generated via alternative splicing, are nuclear factors harboring two ZF domains. Within the hematopoietic system, Evil expression is restricted to long-term and short-term hematopoietic stem cells (HSCs), and under normal conditions, is thereafter during hematopoiesis transcriptionally silent ${ }^{4}$. Activation of the locus, via either chromosomal rearrangement ${ }^{5}$ or by proviral insertion 6,7 , results in marked overexpression of the protein isoforms ${ }^{8}$.

Within hematopoietic tumors, the association between $3 q 26$ rearrangements and myeloid disease is very high: overexpression of EVI1 is virtually never seen in non-myeloid leukemias or lymphomas. The underlying reason for this specific relationship is unknown, but suggest two possibilities: (i) that myeloid cells are particularly susceptible to the transforming effect of EVI1, or (ii) that the overexpression of EVI1 in hematopoietic stem/ progenitor cells (HSPCs) drives cells into the myeloid lineage.

While a number of different mechanisms of action for EVI1 have been suggested (reviewed in ref. ${ }^{9}$ ), it is still unclear how it contributes to leukemogenesis. This is in large part due to the lack of model systems that allow studying the mechanism of EVI1-associated leukemogenesis (reviewed in ref. ${ }^{9}$ ). Experimental efforts to overexpress Evil in mouse models have yielded mixed results ${ }^{10-14}$. These disparate results suggest that EVI1induced disease is difficult to model in the mouse, perhaps due to technical issues; they also suggest that EVI1 by itself is not sufficient to induce neoplastic disease. While Yamazaki et al. ${ }^{14}$ previously published an EVI1 transgenic model that genetically mimics the 3q26 human leukemias and underlined the significance of GATA2 enhancer, we report the development of the first Evil-inducible system that allowed us to uncover a mechanism behind EVI1-associated leukemogenesis.

Using this system, Evi1 induction by doxycycline (DOX) causes a massive perturbation of hematopoietic homeostasis, expanding HSCs, suppressing erythropoiesis and lymphopoiesis, and creating a myeloid-skewed phenotype. Our studies provide insight into the molecular mechanism, as they demonstrate that the myeloid skewing is dependent on DNA binding by EVI1; we further show that EVI1 binds to and upregulates Spil, encoding PU.1, a master regulator of myelopoiesis. Furthermore, the myeloid skewing is dependent on expression of PU.1. These findings support a model wherein EVI1 acts via PU.1 to push hematopoietic HSPCs towards the myeloid lineage, with concomitant suppression of erythropoiesis and lymphopoiesis; this myeloid expansion, eventually progresses to AML.

\section{Results}

Establishment of a mouse model for $3 q 26$ translocations. To mimic the effects of rearrangements at 3q26 in AML, we created a tetracycline (Tet)-inducible allele of Evi1 (termed Evi1 ${ }^{T O}$ ) in the mouse by inserting seven Tet operons within the first exon (Fig. $1 \mathrm{a}^{15}$ ), allowing for the induction of all three isoforms of Evi1 ${ }^{8,9}$. Evi1 ${ }^{T O / T O}$ mice are viable and fertile without phenotype indicating that the allele functions normally in the uninduced state. Through genetic crossings, we then introduced the ubiquitously-expressed ${ }^{16-18}$ reverse-Tet transactivator (rtTA) allele (Rosa26 ${ }^{\text {rtTA/rtTA; }}$; ref. ${ }^{19}$ ).

To assess whether Evil can be induced in vivo, we tested

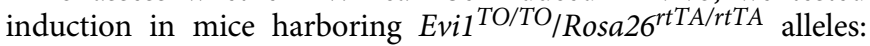
treatment of these mice with DOX results in dramatic upregulation of the major Evil transcript in hematopoietic cells to levels seen in leukemic cells (Fig. 1b); two other mRNA transcripts, encoding shorter isoforms, were also upregulated by DOX (Supplementary Figure 1); however, the longer, MDS1EVI1 mRNA transcript, (encoding PRDM3, the PR-domaincontaining isoform), was not upregulated (Fig. 1b). This accurately models the pattern of MECOM expression commonly seen in human AML with 3q26 rearrangements, in which the EVI1 isoforms are upregulated, but typically not the longer, MDS1-EVI1/PRDM3 isoforms; this particular expression pattern is associated with the poorest prognosis ${ }^{20}$.

To assess the effect of EVI1 overexpression on hematopoiesis in a context that mimics human myeloid malignancy, in which the oncogene-expressing cells are mixed in with normal hematopoietic cells, we performed competitive transplantations in which the oncogene-expressing bone marrow was mixed 1:1 with WT bone marrow (Fig. 1c); for examination of the short-term effects of EVI1 overexpression, we also fed DOX chow directly to the Evi1 TO/TO/Rosa26rtTA/rtTA mice themselves (Fig. 1d).

In the competitive transplantation model, to allow each donor origin to be traced by flow cytometry, the WT competitor was marked with UBC-GFP, while Evi1 TO/TO/Rosa26 $6^{\text {rtTA/rtTA }}$ cells were of CD45.2/GFP-negative origin; transplants were performed into lethally irradiated CD45.1/GFP-negative recipients. For a control cohort, we transplanted Evi1 ${ }^{W T / W T} /$ Rosa26 $^{W T / W T}$ CD45.2/GFP-negative bone marrow cells instead of the Evi1 ${ }^{T O} /$ TO/Rosa26 $6^{\text {rtTA/rtTA }}$ cells together with GFP-positive WT cells (Fig. 1c). We documented that the stem cell and progenitor populations from the bone marrow of $E v i 1^{T O / T O} / R_{0 s a 26^{r t T A} / r t T A}$ donors did not differ from that of the control just prior to transplantation (Supplementary Figure 2A). Recipients were analyzed for the engraftment of donors' BM 4 weeks post transplantation and prior to EVI1 induction, which showed that the two donors (GFP-positive and GFP-negative) were no different, both in control recipients and those receiving Evi1 ${ }^{T O / T O} /$ Rosa26 $^{\text {rtTA/rtTA }}$ cells (Supplementary Figure 2B, left). After confirming successful engraftments, recipients were placed on DOX chow to induce Evil. After 10 weeks, we analyzed cells in peripheral blood and bone marrow. The mice appeared healthy, and CBCs revealed no cytopenias (not shown). We performed flow analysis of marrow and blood, identifying Evi1 ${ }^{\text {TO/TO/Rosa } 26^{r t T A / r t T A}}$ cells by lack of GFP, and engraftment ratio was determined, which revealed a significant (more than twofold) expansion of the EVI1-overexpressing compartment (Supplementary Figure 2B, right).

EVI1 overexpression suppresses erythropoiesis. Analysis of the erythroid lineage in competitively transplanted mice at 2, 6, and 10 weeks post induction showed a deficiency in the ability of the EVI1-overexpressing cells to effectively contribute to erythropoiesis (Fig. 2a-c). At the 6 and 10 weeks post-induction time points, the number of EVI1-overexpressing Ter119-positive nucleated erythroid cells within the bone marrow was significantly lower than the number of GFP-negative WT cells in control transplant recipients (Fig. 2a). In the peripheral blood of chimeric mice harboring Evi1 TO/TO, Rosa26rtT/rtTA cells, EVI1-overexpressing reticulocyte, and erythrocyte numbers were significantly lower than control at every time point (Fig. 2b, c). During the same time period, there was no difference in platelets (Fig. 2d), or megakaryocytes between EVI1-overexpressing and wild-type GFP cells.

To understand the etiology of the lower numbers of erythroid cells in the EVI1-overexpressing compartment, we quantitated apoptosis and proliferation in erythroid progenitors using flow 
a

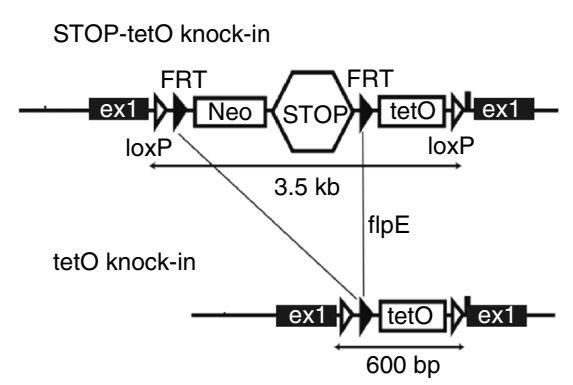

b

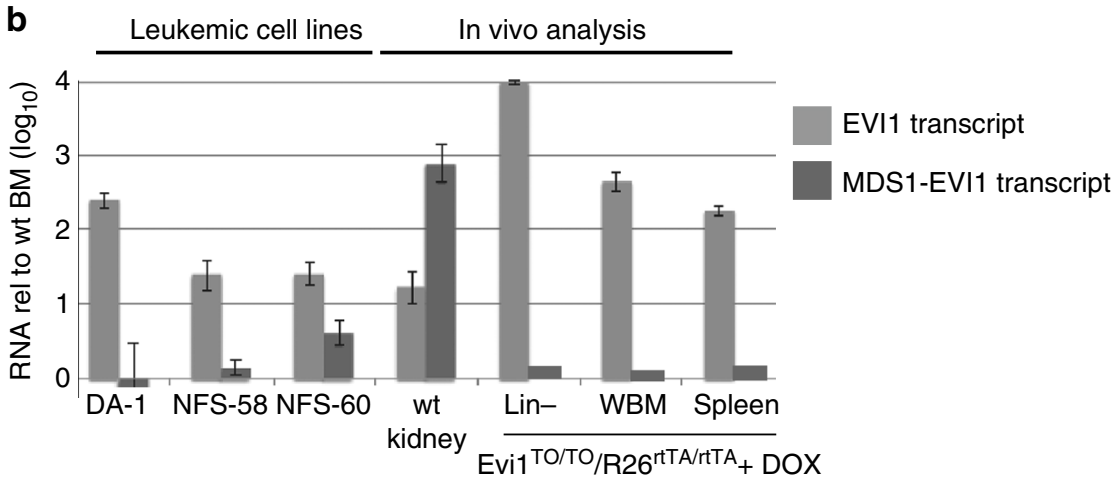

C
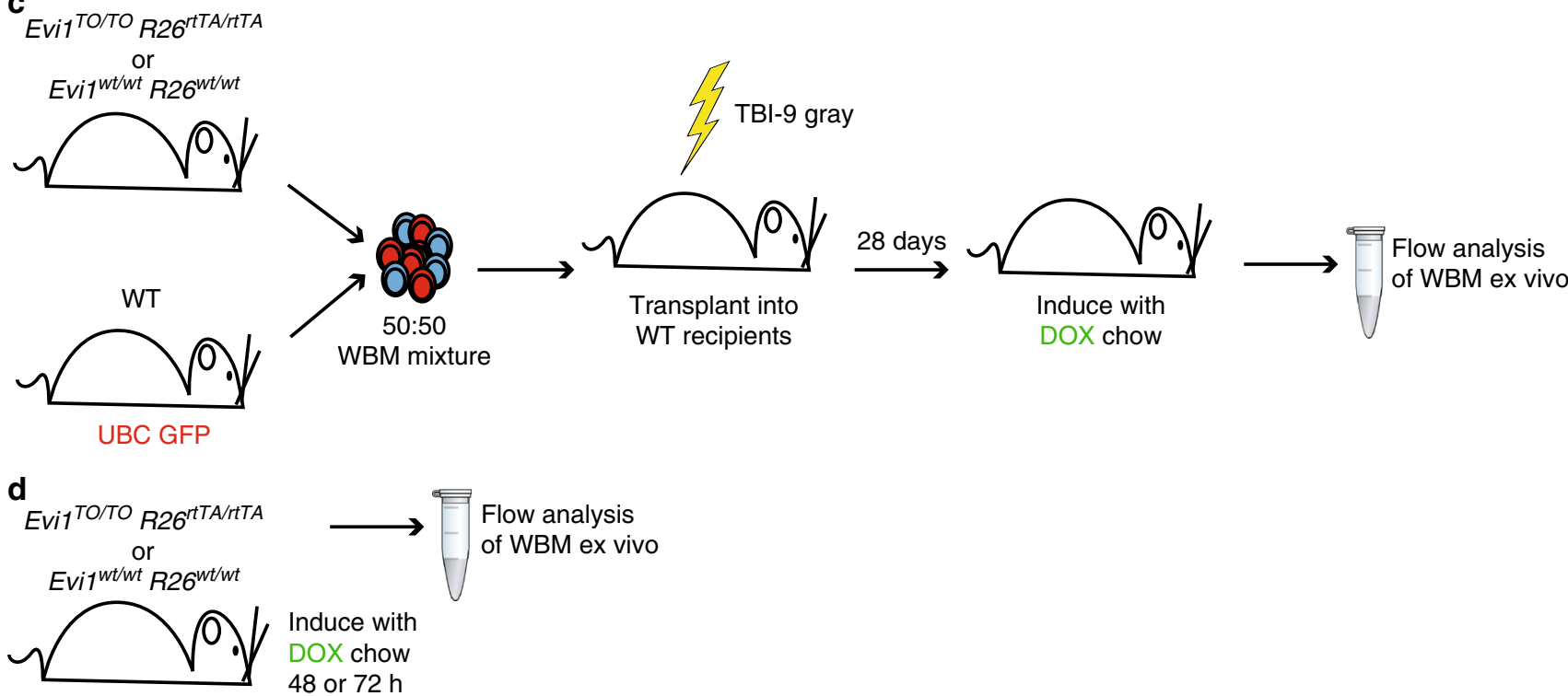

Flow analysis of WBM ex vivo

Fig. 1 Evit TO mouse model for 3q26-rearranged myeloid malignancies. a Schematic diagram of the Neo-Stop-Tet Operon (NSTO) construct from Tanaka et al. ${ }^{63}$ that was inserted into the endogenous Evi1 locus by homologous recombination. The construct consists of a neomycin resistance gene and transcriptional Stop cassette flanked by FRT sites, followed by seven Tet operons in succession and a minimal CMV promoter. Following homologous recombination in embryonic stem cells, the Neo-STOP cassette was removed by induction of flpE recombinase. Black triangles represent FRT sites, and white triangles represent loxP sites. $\mathbf{b}$ Reverse transcription-quantitative polymerase chain reaction (RT-qPCR) analysis of RNA from cultured leukemic cell lines bearing a provirally-activated EVI1 allele (left-hand columns), or RNA from mouse tissues from wild-type (kidney, an organ with relatively high levels of expression of both Evi1 and Mds1-Evi768), and from DOX-induced Evi1 TO, Rosa26rtA mice. Shown is quantitation of Evi1 and Mds1-Evi1 transcripts. Data is normalized to EVI1 RNA levels in WT bone marrow; $\log _{10}$ scale. c Schematic of competitive transplant long-term induction model. CD45.2 whole bone marrow from either Evi1 ${ }^{T O}$ or a WT control with the indicated genotypes was mixed at a 1:1 ratio with WT UBC-GFP-positive bone marrow and transplanted into lethally irradiated recipient mice. Mice were placed on DOX chow at 4 weeks post transplant and analyzed 2, 6, or 10 weeks post induction. Long-term induction experiment was done twice (set $A, n=24$, set $B, n=24$ ). Each time point of each set had 8 mice $(W T, n=4, E V I 1, n=4)$. (Total $n=48$ ). d Schematic of endogenous short-term induction model. Mice were induced with DOX chow for 48 or $72 \mathrm{~h}$ and whole bone marrow was harvested for ex vivo analysis

cytometry; these studies revealed a sixfold increase in apoptosis within the erythroblasts relative to WT cells (Fig. 2e), and a marked drop in the number of, and proliferation of, both proerythroblasts and erythroblasts relative to WT (Fig. 2f, g).

In summary, EVI1 overexpression has a dramatic effect on erythropoiesis and this is mediated by both an increase in apoptosis and a decrease in proliferation.

EVI1 overexpression suppresses lymphopoiesis. We examined the bone marrow at $2-10$ weeks post induction for changes in $\mathrm{T}$ and B cells; we noted significantly lower numbers of EVI1overexpressing B-cell lineage cells within the bone marrow at 6 and 10 weeks time points (Fig. 3a). While the number of Evil ${ }^{T O / T O} /$ Rosa $26^{r t T A / r t T A} \mathrm{~T}$ cells in the bone marrow was not significantly different from the WT control, examination of blood for lymphocytes showed a marked diminution in both $\mathrm{T}$ and $\mathrm{B}$ cells from the EVI1-overexpressing compartment: at 10 weeks post induction, EVI1 overexpression caused a decrease in peripheral $\mathrm{T}$ cells from $\approx 1800$ cells per $\mu \mathrm{l}$ to $\approx 750$ cells per $\mu \mathrm{l}$, and nearly eliminated the peripheral B cells completely (Fig. $3 \mathrm{~b}$ ). This was associated with a two to threefold increase in the number of apoptotic bone marrow $\mathrm{T}$ and $\mathrm{B}$ cells (Fig. $3 \mathrm{c}$ ).

We found that the number of $\mathrm{T}$ cells in the marrow was low, which is to be expected: T cells are derived from HSCs in the bone marrow, but at an early stage, migrate from the bone marrow to the thymus, where they complete their development. To better assess the effect of EVI1 overexpression on $\mathrm{T}$ cells, we enumerated $\mathrm{T}$ cells in the thymus at the double (CD4/CD8)positive stage, and at the CD4 or CD8 single-positive stage. This 
a

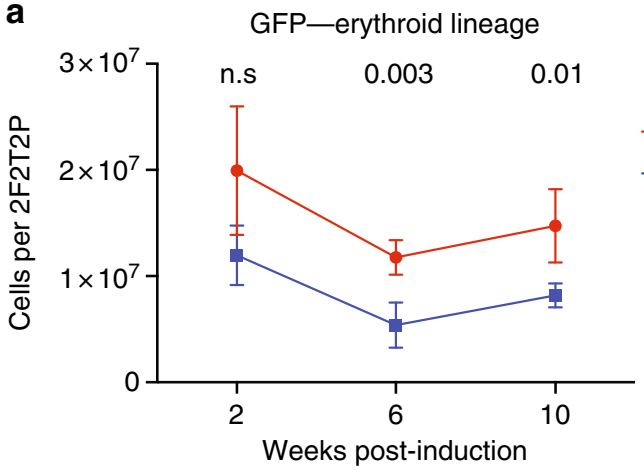

C

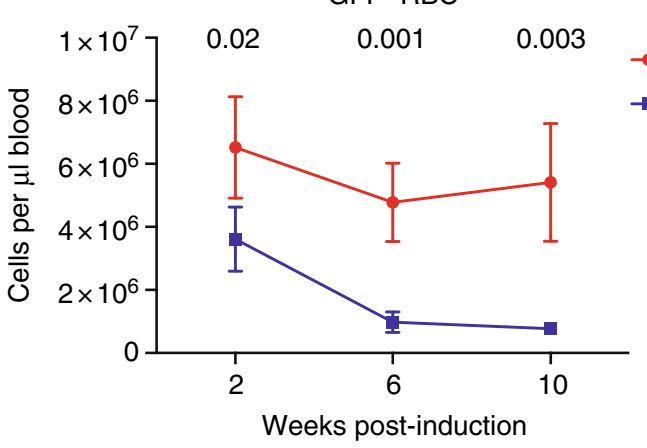

b

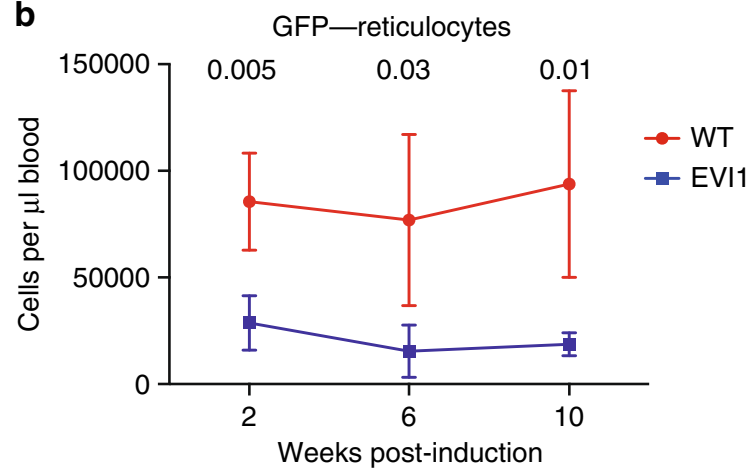

d

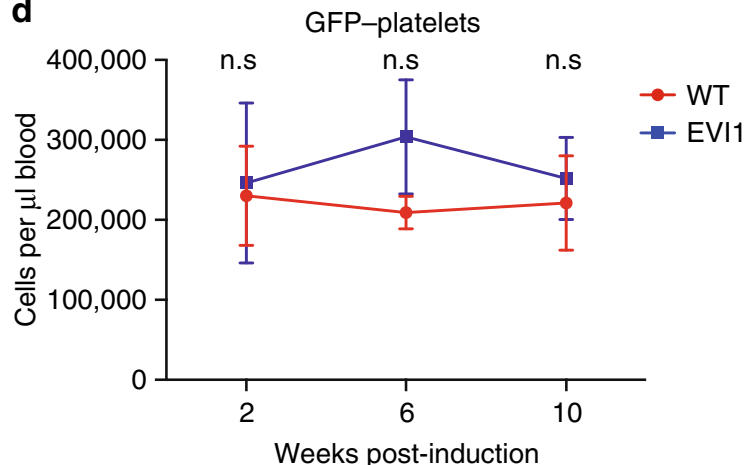

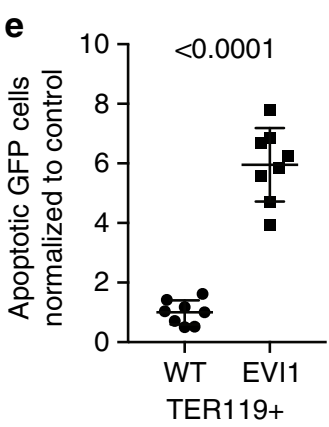
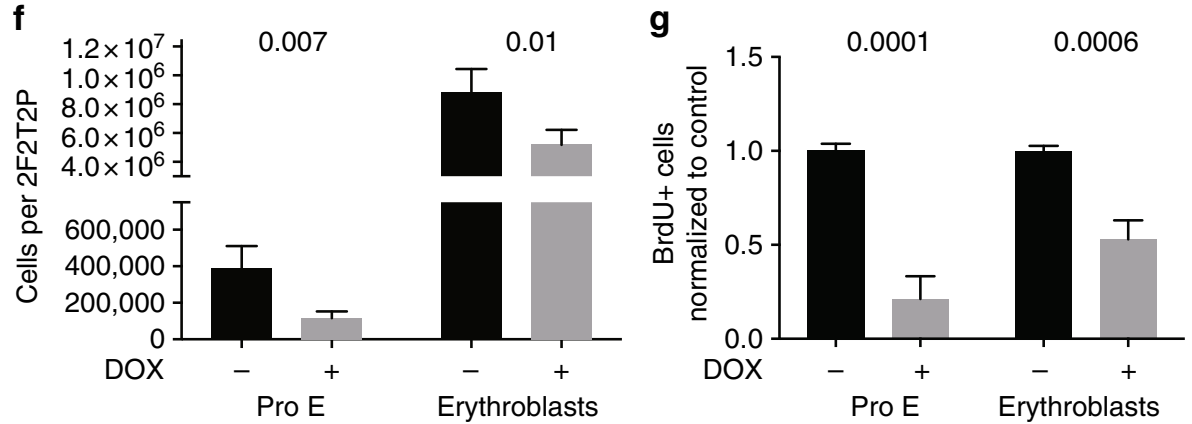

Fig. 2 EVI1 overexpression suppresses erythropoiesis. a Quantification of erythroid (TER119+) cells overexpressing EVI1 (GFP-) in bone marrow of competitively transplanted recipients harvested 2, 6, or 10 after DOX induction. (WT $n=4$ mice, EVI1 $n=4$ mice at each time point, total $n=24$ ). b-d Numbers of donor-derived reticulocytes (GFP-, Ter119+, CD71+), erythrocytes (GFP-,Ter119+, CD71-), and platelets (GFP-, low SSC/FSC, CD41 $+)$ as indicated at 2, 6, and 10 weeks post induction per microliter of peripheral blood of mice competitively transplanted with DOX-induced EviT ${ }^{T O}$, Rosa26rtA mice or WT cells. (WT $n=4$ mice, EVI1 $n=4$ mice at each time point, total $n=24$ ). e Quantification of apoptotic (annexin-v+ 7AAD-) erythroid (TER119+) cells overexpressing EVI1 (GFP-) or WT WBM of competitively transplanted recipients, expressed relative to WT GFP- cells from the control transplant, harvested 10 weeks after DOX induction. Each dot represents an individual recipient mouse. (WT $=8$ mice, EVI1 $=8$ mice). f, $\mathbf{g}$ Quantification of total (f) and BrdU-positive (g) proerythroblasts (TER119+low cKit+), and erythroblasts (TER119+ CD71+) in WBM of EviT ${ }^{\text {TO/TO }}$ Rosa26rtA/rtTA mice harvested $48 \mathrm{~h}$ with or without DOX induction. (WT $n=4$, EVI1 $n=4$ ). For all panels, error bars represent standard deviation; $p$ values calculated with Student's $t$-test

revealed a marked diminution of double-positive cells relative to control but little change in CD4-positive $\mathrm{T}$ cells (Fig. 3d). Interestingly, there was an increase in CD8-positive $\mathrm{T}$ cells (Fig. 3d). To further assess these changes, we assessed apoptosis and proliferation (Fig. 3e, f) and found increased apoptosis only in the double-positive compartment, but decreased proliferation in both the double-positive and CD8 single-positive population.

EVI1 overexpression expands myelopoiesis. At 2 weeks post induction, EVI1-overexpressing and control bone marrows showed the same number of myeloid cells (GFP-negative, CD11bpositive (Fig. 4a) or Ly6G/C-positive (Fig. 4b)). However, at 6 and 10 weeks post induction, there was marked expansion of the
EVI1-overexpressing myeloid compartment, from $\approx 7.5 \mathrm{e} 6$ cells/ $2 \mathrm{~F} 2 \mathrm{~T} 2 \mathrm{P}$ to $\approx 30 \mathrm{e} 6$ cells/2F2T2P for $\mathrm{CD} 11 \mathrm{~b}+$ cells, and from $\approx 12 \mathrm{e} 6$ cells/2F2T2P to $\approx 36 \mathrm{e} 6$ cells/2F2T2P for $\mathrm{LY} 6 \mathrm{G} / \mathrm{C}+$ cells (Fig. 4a). We also quantified HSCs and myeloid progenitors using flow cytometry at 10 weeks post induction; The expansion of lineage-negative, $c-K i t+$, Sca-1+ (LSK cells; approximately twofold) was not significant $(p>0.05)$, but both CMPs and granulocyte-monocyte progenitors (GMPs) were significantly increased (Fig. 4c). By performing colony-forming assay, we found that myeloid colony-forming cells were over 2.5-fold increased relative to controls (Fig. 4d). By flow-based analysis, monocytes/macrophages and granulocytes within the marrow were increased (Fig. 4e). However, blood monocytes were unchanged, and granulocytes were increased but not as 


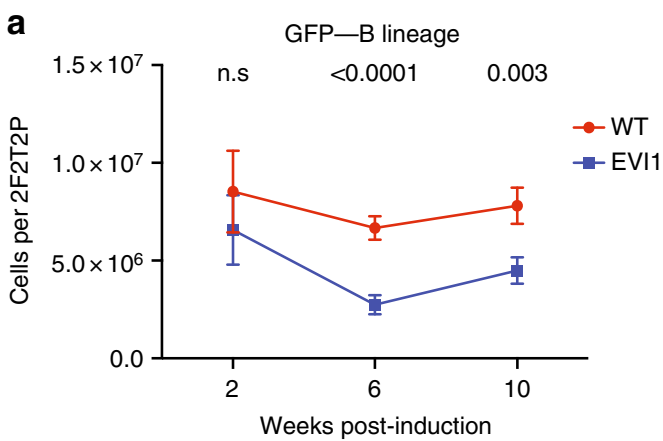

d

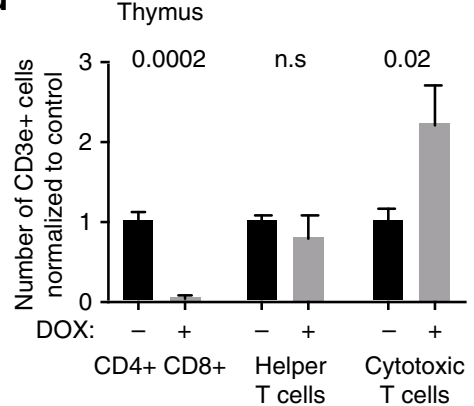

b

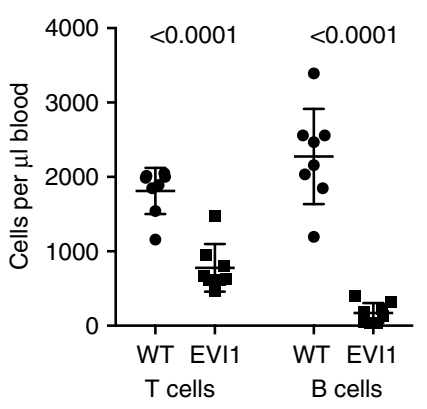

c

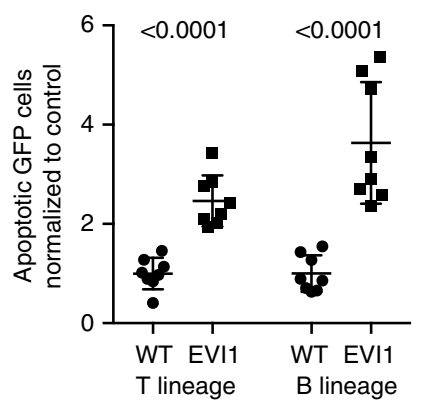

e

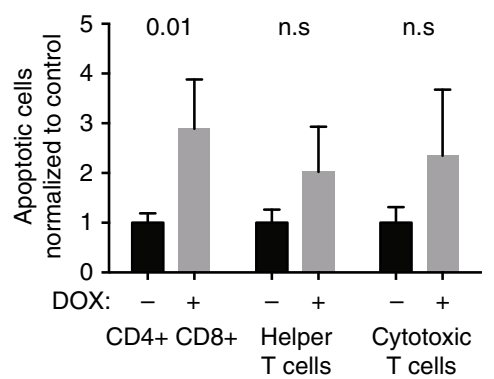

f

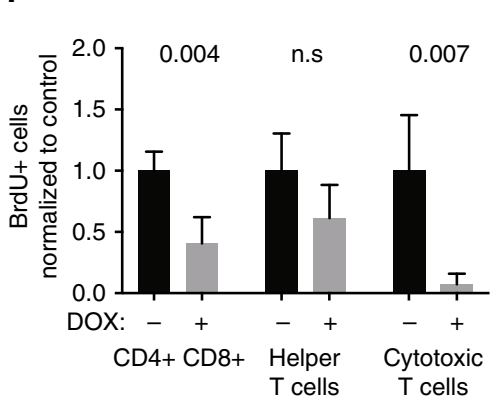

Fig. 3 EVI1 overexpression suppresses lymphopoiesis. a Quantification of B lymphocytes (B220+) cells overexpressing EVI1 (GFP-) and WT control (GFP-) in WBM of competitively transplanted recipients harvested 2, 6, or 10 after DOX induction. (WT $n=4$ mice, EVI1 $n=4$ mice at each time point, total $n=24)$. b Circulating donor-derived T cells (GFP-, CD3e+, CD11b-) and B cells (GFP-, CD19+, CD11b-) in competitively transplanted mice at 10 weeks post induction. Each time point (WT $n=8$ mice, EVI1 $n=8$ mice). c Quantification of apoptotic (annexin-V+, 7AAD-) T lymphocytes (CD3e+) and B lymphocytes (B220+) overexpressing EVI1 (GFP-) compared to WT (GFP-) in WBM of competitively transplanted recipients harvested 10 weeks after DOX induction. Each dot represents an individual recipient mouse. (WT $n=8$ mice, EVI1 $n=8$ mice). d Quantification of CD4/CD8 double-positive cells (CD3e+, CD4+, CD8a+), helper T cells (CD3e+, CD4+, CD8a-), and cytotoxic T cells (CD3e+, CD4-, CD8a+) in thymus of Evi7 ${ }^{\text {TO/TO }}$

Rosa26rtA/rtTA mice harvested $48 \mathrm{~h}$ with or without DOX induction. (WT $n=4$, EVI1 $n=4$ ). e Quantification of apoptotic (annexin-v+ 7AAD-) CD4/ CD8 double-positive cells (CD3e+, CD4+, CD8a+), helper T cells (CD3e+, CD4+, CD8a-), and cytotoxic T cells (CD3e+, CD4-, CD8a+) in thymi of Evi1 ${ }^{\text {TO }} / T_{O}$ Rosa26rtA/rtTA mice harvested $48 \mathrm{~h}$ with or without DOX induction. (WT $n=4$, EVI1 $n=4$ ). $\mathbf{f}$ Quantification of BrdU+ CD4/CD8 double-positive cells (CD3e+, CD4+, CD8a+), helper T cells (CD3e+, CD4+, CD8a-), and cytotoxic T cells (CD3e+, CD4-, CD8a+) in thymi of EviT TO/TO Rosa26rtTA/rtTA mice harvested $48 \mathrm{~h}$ with or without DOX induction. (WT $n=4$, EVI1 $n=4$ ). For all panels, error bars represent standard deviation; $p$ values calculated with Student's $t$-test

significantly as in the marrow. Analysis of annexin- $\mathrm{V}$ revealed no increase in apoptosis of the marrow myeloid cells (Fig. 4f). We also assessed the composition of the LSK compartment in the marrows, which contain HSCs and multipotent progenitors (MPPs) using the surface marker phenotype described and detailed by Cabezas-Wallscheid et al. ${ }^{21}$ (Supplementary Figure 2C, Supplementary Table 1). We found that Evil expression expands HSCs, multipotent MPP2s, and the myeloid-biased MPP3 populations, while reducing the erythroid-biased MPP4 populations; no change in MPP1 was seen (Supplementary Figure 2D).

In summary, these data indicate that Evil overexpression induces myeloid expansion via a proliferative and a survival advantage; increased maturation/differentiation from uncommitted progenitors at a higher rate may also play a role.

EVI1 overexpression results in AML. To determine if chronic overexpression of EVI1 might result in leukemia, we aged a cohort of five mice, competitively transplanted with a 1:1 mix of WT bone marrow cells:Evi1 ${ }^{T O / T O} /$ Rosa26rtTA/rtTA cells, chronically on DOX chow to maintain high-level expression of EVI1. All five mice succumbed, becoming moribund at 90-119 days of DOX treatment (median $=118$ days; Fig. $5 \mathrm{a} ; p=0.002$; Fig. $5 \mathrm{a}$ ); flow and morphological analysis revealed AML in all mice analyzed, with the bone marrow replete with blast forms (Fig. 5b), which, by immunophenotyping by flow cytometry, were positive for Mac1 (CD11b) (Fig. 5c). The peripheral blood revealed severe anemia (Fig. 5d).

EVI1 myeloid-biased phenotype depends on DNA binding by EVI1. The data shown above indicate that Evil overexpression results in suppression of erythroid and lymphoid cells, and expansion of myeloid cells. Since EVI1 harbors two DNA-binding zinc-finger domains (Supplementary Figure 1G; refs. ${ }^{22,23}$ ) and can modulate the transcription of target genes via binding in cis (e.g., refs. ${ }^{15,24}$ ), we wished to determine if the myeloid skewing phenotype induced by EVI1 was dependent on its binding to DNA using two EVI1 mutants (Fig. 6a): EVI1-R205N, which lacks DNA binding via ZF domain $1^{8}$ and EVI1-R769C, which lacks DNA binding via $\mathrm{ZF}^{25}$. However, rather than testing these in our mouse model that would have entailed the development of two new mouse strains, instead we utilized EML cells $^{26}$, a hematopoietic progenitor cell line that does not express Evi1. Previous analysis of EML cells ${ }^{27,28}$ indicated that Sca- 1 provides an excellent marker for the lineage proclivity of these progenitor cells, with Sca-1 ${ }^{\text {lo }}$ cells showing high GATA-1 expression and being poised for erythroid differentiation, and Sca- $1^{\text {hi }}$ cells expressing high PU.1 expression and being poised for myeloid differentiation 27,28 . To determine if Sca-1 upregulation depended on the action of EVI1 on downstream targets, we tested the two EVI1 DNA-binding mutants (Fig. 6a, EVI1R205N and EVI1-R769C) in EML cells for their ability to 

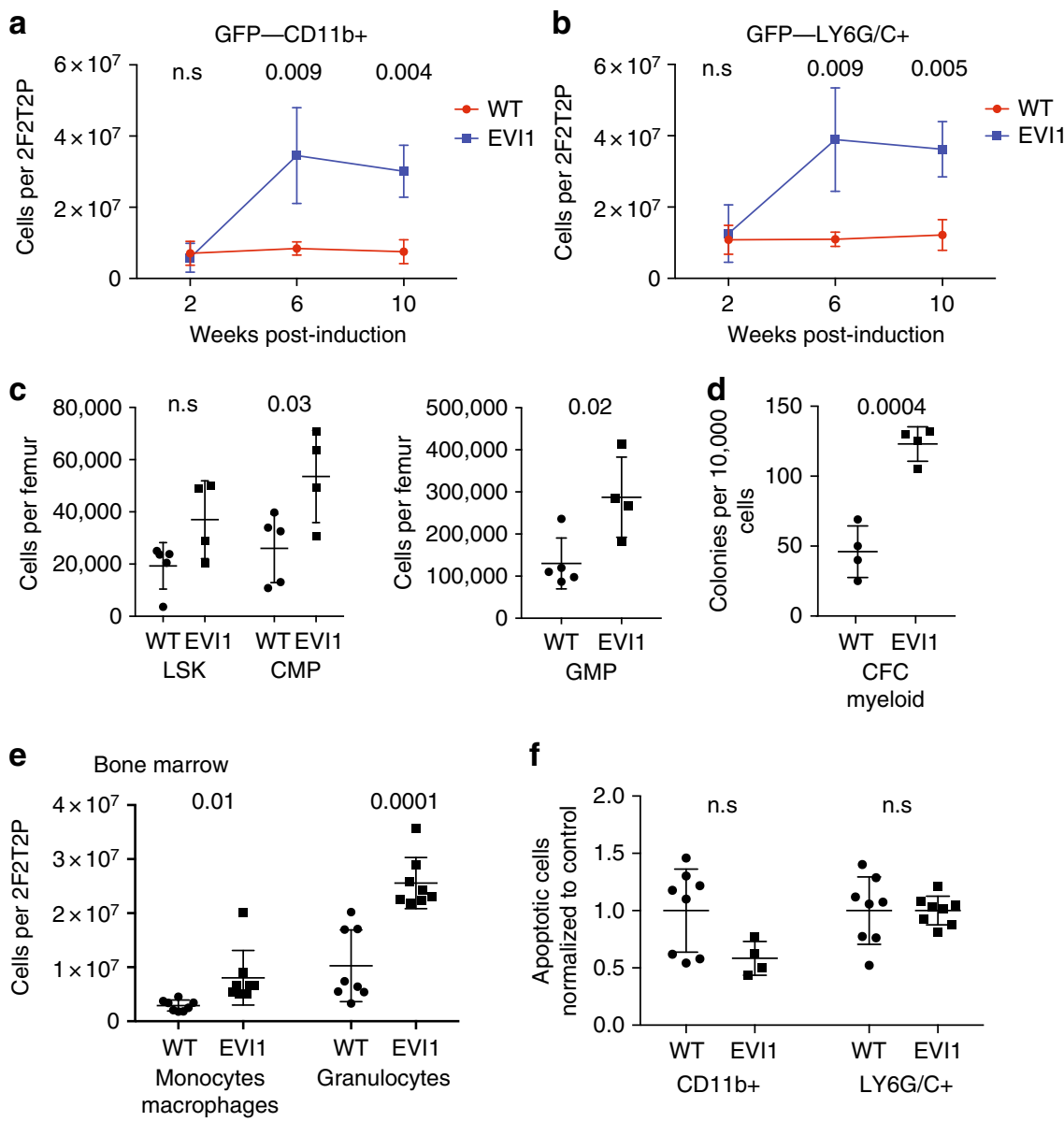

$\mathbf{f}$

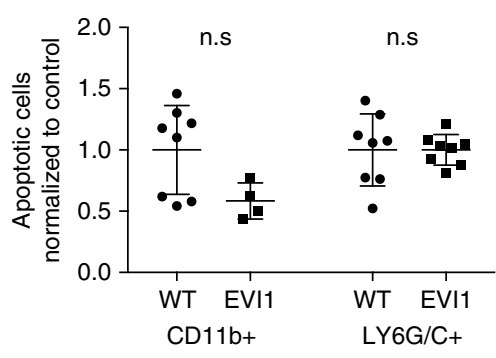

Fig. 4 EVI1 overexpression expands myelopoiesis. a Quantification of GFP-, CD11b+ myelocytes, either overexpressing EVI1 or WT as indicated in WBM of competitively transplanted recipients, harvested 2, 6, or 10 weeks after DOX induction. (WT $n=4$ mice, EVI1 $n=4$ mice at each time point, total $n=24)$. b Quantification of GFP - LY6G/C + myelocytes, either overexpressing EVI1 or WT in WBM of competitively transplanted recipients harvested 2, 6 , or 10 weeks after DOX induction. (WT $n=4$ mice, EVI $n=4$ mice at each time point, total $n=24$ ). c Flow cytometry analysis of progenitor populations from recipient mice after 10 weeks of DOX treatment. Data represents mean number of cells per femur for individual mice. (WT $n=5$, Evi1 $+n=4)$.

d Relative numbers of GFP- CFC-myeloid (G-, M and GM-CFC) in bone marrow of competitively transplanted mice 10 weeks post induction, comparing the Evi1 TO/TO Rosa26rtTA/rtTA:WT to WT:WT transplants as indicated. (WT $n=8$, EVI1 $n=8$ ). e Quantification of GFP- monocyte/macrophage (nucleated, $\mathrm{CD} 11 \mathrm{~b}+, \mathrm{Gr} 1-$ ) or GFP- granulocytes (nucleated, $\mathrm{CD} 11 \mathrm{~b}+, \mathrm{Gr} 1+$ ) as determined by imaging flow cytometry in bone marrow of competitively transplanted mice, comparing EviT TO/TO Rosa26rtA/rtTA donor cells to WT donor cells, 10 weeks post induction. (WT $n=8$, EVI1 $n=8$ ). f Quantification of GFPapoptotic (annexin-V+ 7AAD-) CD11b+ myelocytes (left) and LY6G/C+ myelocytes (right), comparing WT to EVI1-overexpressing cells in WBM of competitively transplanted recipients harvested 10 weeks after DOX induction. Each dot represents an individual recipient mouse. (WT $n=8, E V I 1 n=8)$. For all panels, error bars represent standard deviation; $p$ values calculated with Student's $t$-test

upregulate Sca-1. Both of these mutations abrogated the upregulation of Sca-1 (Fig. 6b), indicating that the myeloid skewing phenotype requires both ZF1 and ZF2 domains and that the action of EVI1 on downstream target genes is an essential mechanistic link. To investigate this further, we assessed the expression of five key hematopoietic regulators in vectortransduced EMLs, compared to EVI1-expressing EMLs, as well as those expressing ZF mutants, specifically focusing on the Sca$1^{\text {hi }}$ population (Fig. 6c). These regulators were Spil, encoding PU.1, a major regulator of the lineage choice between erythroid and myeloid cells ${ }^{29,30}$; Cebpe, encoding C/EBP- $\varepsilon$, essential for terminal myeloid maturation ${ }^{31}$; Cebpg, encoding CEBP- $\gamma$, essential for NK cell development ${ }^{32}$ and which interacts with CEBP- $\alpha$, a major regulator of myelopoiesi ${ }^{33} ; \mathrm{Lmo}^{2}$, encoding a transcriptional regulator and facilitator of DNA replication ${ }^{34}$ that is essential for adult hematopoiesis ${ }^{35}$; and Gfilb, which is required for erythropoiesis and thrombopoiesis ${ }^{36}$. We found significant changes in three of these: Spil (up twofold), Cebpg (down 50\%), and Lmo2 (up 10-fold). For Spil, Cebpg, and Lmo2,
EVI1-induced changes in expression were dependent on DNA binding, since point mutations in ZF1 or ZF2 abrogated the effect (Fig. 6c).

EVI1 myeloid-biased phenotype is dependent on Spi1. The transcription factor PU.1 is a master regulator of early myelopoiesis, with highest levels being expressed in CD11bpositive myeloid cells, including macrophages, monocytes, and immature granulocytes, and lower levels in B lymphocytes, while erythroid cells lack expression ${ }^{37}$. We posited that activation of Spil by EVI1 could help explain the expansion of the myeloid compartment in EVI1-overexpressing bone marrow. Thus, we decided to study the link between EVI1, Spi1/PU.1, and myeloid skewing.

To confirm the upregulation of Spil by EVI1 overexpression in HSPCs in vivo, we assessed changes in Spil gene expression following EVI1 induction with DOX in our Evi1TO/Rosa26 rtTA mice. We found that EVI1 upregulates Spil expression in HSCs and progenitors (LSK) and in GMPs (Fig. 6d) suggesting that 
a

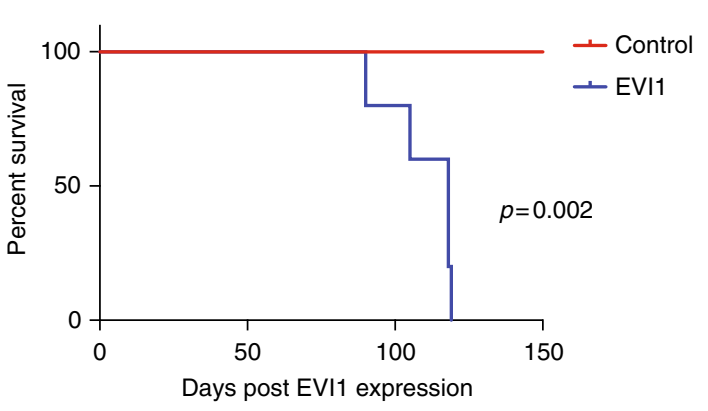

C

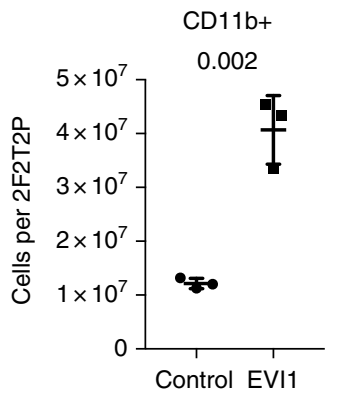

d

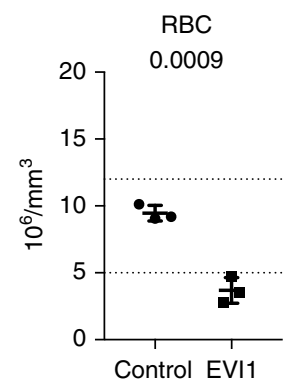

b

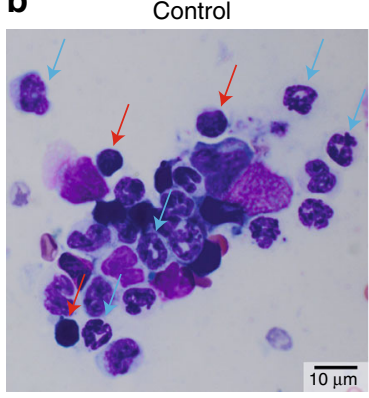

$\mathrm{B} 220+$
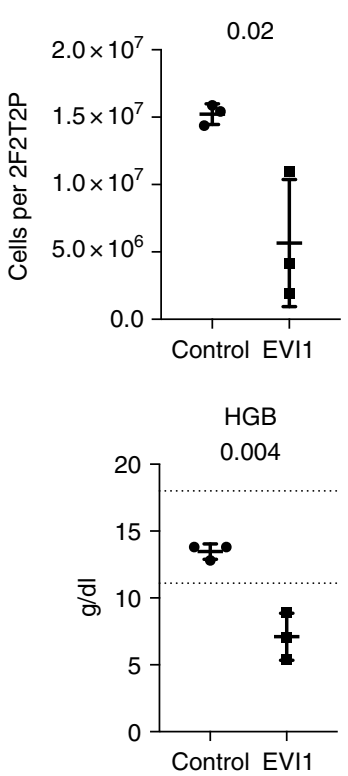
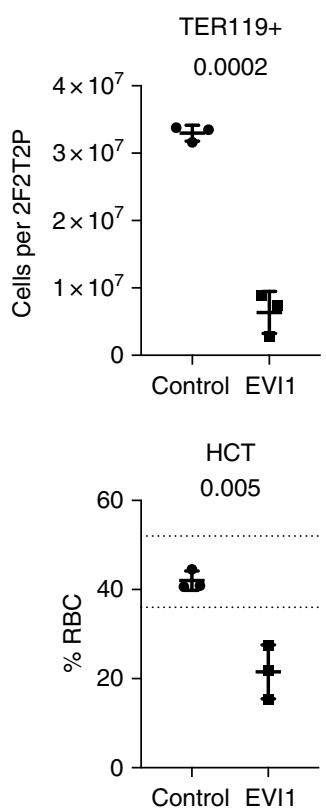

Fig. 5 EVI1 overexpression results in AML with intermediate latency. a Kaplan-Meier curve showing survival of CD45.1 mice that were transplanted with WT:EviT TO/TO, R26R rtTA/rtTA at a 1:1 ratio with and without DOX induction. Mice were harvested when morbidity presented (survival range $=$ 90-119 days, median $=118$ days, $n=5$ ) b Representative Wright-Giemsa-stained bone marrow brush smears from CD45.1 mice that were transplanted with WT:Evi7 ${ }^{T O} / T O, R 26 R^{\text {rtTA/rtTA }}$ at a 1:1 ratio without (left) or with (right) DOX induction at the day of harvest. Mice were harvested when morbidity presented. Blue arrows, maturing myeloid forms (metamyelocytes and bands); red arrows, erythroblasts; yellow arrows, leukemic blasts (scale bars: $10 \mu \mathrm{m}$ ). c Quantification of CD11b+ cells (left), B220+ cells (middle), or TER119+ cells (right) using flow cytometry analysis on WBM of CD45.1 mice that were transplanted with WT:Evi1 TO/TO, R26RrtTA/rtTA at a 1:1 ratio with and without DOX induction at the day of harvest. Mice were harvested when morbidity presented $(n=3)$. d Quantification of red blood cells (RBC), hemoglobin (HGB), and hematocrit (HCT) using complete blood count assay performed on PB from CD45.1 mice that were transplanted with WT:EviT TO/TO, R26R ${ }^{\text {rtTA/rtTA }}$ at a 1:1 ratio with and without DOX induction at the day of harvest. Mice were harvested when morbidity presented $(n=3)$. For all panels, error bars represent standard deviation; $p$ values calculated with Student's t-test

EVI1 increases the expression of the key myeloid gene Spil in early hematopoietic and myeloid progenitor cells. We then analyzed Spil expression in sub-compartments within the LSKs: we observed strong upregulation of Spil within the lineagenegative/Sca- ${ }^{\text {hi }}$ compartment, as well as in long-term HSCs (LTHSCs; Fig. 6e).

Our data in the EML cell system (Fig. 6b) implies that EVI1 upregulates PU.1, leading to increase in myeloid poised Sca- 1 hi cells. This predicts that knockdown of PU.1 in EVI1-transduced cells would lead to decreased percentage of Sca-1 high cells. We introduced Spil-specific shRNA constructs specific into EMLEVI1 cells, and quantitated both Sca-1 and Spi1; the results show a strong positive correlation between Sca-1 expression and Spi1 expression: the higher the level of PU.1, the greater the percentage of Sca- ${ }^{\text {hi }}$ cells (Fig. $6 \mathrm{f}$ ). These data indicate that EVI1-mediated upregulation of Sca-1, and hence myeloid skewing, depends on Spi1/PU.1.
PU.1 is necessary for EVI1-mediated upregulation of Sca-1. To better understand the relationship between EVI1, PU.1, and Sca1 , we turned to an in vivo system in which PU.1 expression can be followed at the single cell level: we utilized a Spi1gfp allele, in which GFP is driven by the endogenous Spil promoter ${ }^{37}$. We crossed our Evi1 ${ }^{T O}$ and Rosa26rtTA alleles with the Spi1gfp allele and assessed whether DOX-induced upregulation of Evil expression would lead to increased Spi1/PU.1 and Sca-1 expression within the bone marrow.

Bone marrow from DOX-induced $E v i 1^{T O} /$ Rosa $6^{r T T} / S p i l^{g f p}$ mice was isolated at $24 \mathrm{~h}$, and $48 \mathrm{~h}$ post induction and analyzed for GFP and Sca-1 expression. We found a significant upregulation of GFP, from 55 to $70 \%$ of cells by $24 \mathrm{~h}(p=0.02)$ (Fig. $6 \mathrm{~g}$ ). This was followed by a marked increase in Sca-1 expression at 48 h (Fig. 6g, bottom panel).

We wondered whether EVI1 overexpression within early erythroid cells could induce Sca-1 expression. From previous 
a

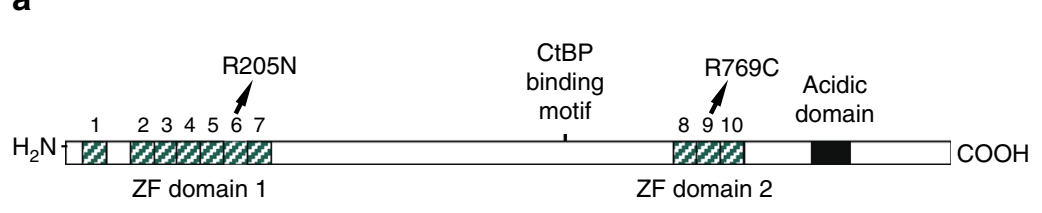

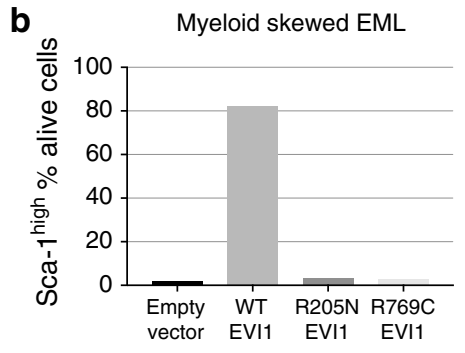

e

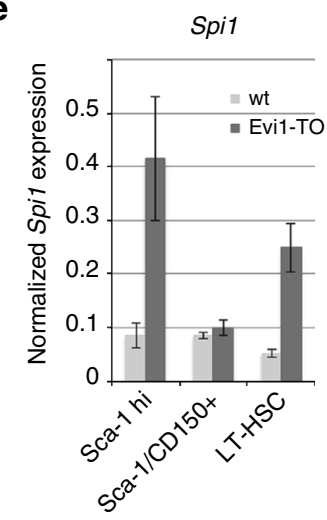

f
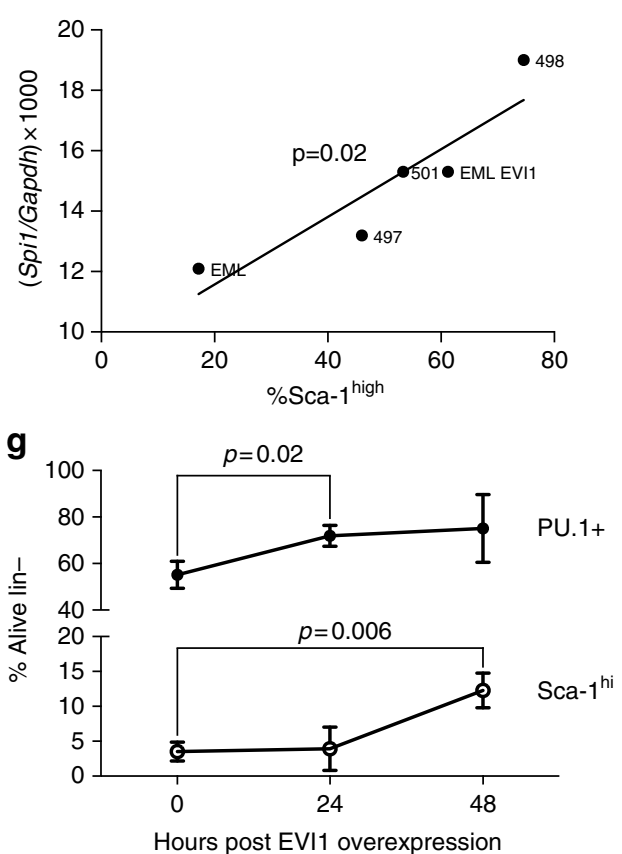

h DOX-

PU.1- sorted
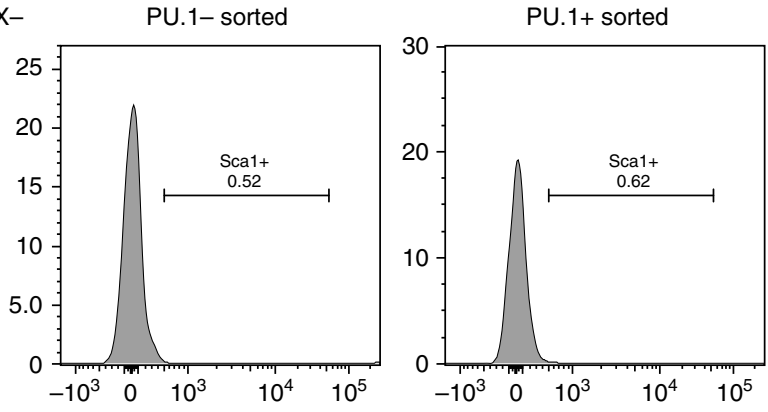

$\mathrm{DOX}+$

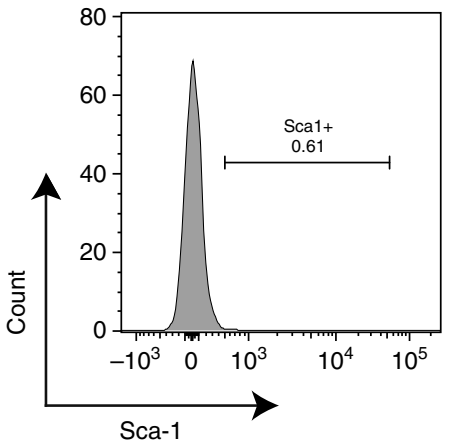

Fig. 6 EVI1-induced myeloid skewing: downstream effectors include PU.1. a Schematic of EVI1 protein domains, documenting the two zinc-finger domains and the location of the two arginine mutations that disrupt ZF1 and ZF2. b Flow cytometric quantitation of Sca- $7^{\text {hi }}$ myeloid-skewed population in EML cells transduced with the indicated retroviral construct, designated on $x$ axis. c RT-qPCR analysis of RNA from sorted Sca- $\rceil^{\text {hi }}$ EML cells transduced with the indicated constructs. Gene names are indicated on the $x$ axis. All qPCR reactions performed in triplicate; displayed values represent the average. Errors bars represent standard deviation between $\mathrm{qPCR}$ reactions. ${ }^{*} p<0.005$. d RT-qPCR data (normalized to Gapdh) showing expression of Spi1 in progenitor populations from Evit ${ }^{T O} / T O_{R 26 r}{ }^{T A}$ /rtTA CD45.2 donor compartments at 10 weeks of induction. e RT-qPCR data (normalized to Gapdh) for Spil expression in sorted bone marrow progenitor populations from either WT mice or EviT TO, R26rtA mice (as indicated) treated with DOX for 72 hrs. $\mathbf{f}$ Scatterplot comparing Spi1 mRNA expression ( $y$ axis) to percent Sca-1 hi cells ( $x$ axis) for five EML cell populations: unmanipulated EMLs ("EML"); EVI1-overexpressing EMLs ("EML EVI1"), and three derivatives of the latter, transduced with either a Spi1-specific shRNA (497, 501) or a control shRNA (498). The $p$ value is calculated using linear regression analysis. $\mathbf{g}$ Flow cytometric analysis for Sca-1 and PU.1 (GFP+; top, percent positive) and Sca-1 (bottom; percent positive) on lineage-subpopulation within WBM harvested from Evi1 TO/TO, Rosa26rtTA/rtTA, Spi1 GFP/GFP mice at 0, 24, or $48 \mathrm{~h}$ after DOX induction $(0 \mathrm{~h} n=3,24 \mathrm{~h}$ $n=3,48 \mathrm{~h} n=3$ ). $\mathbf{h}$ Flow cytometric analysis for Sca-1 in Lin-Sca-1-cKit-PU.1GFP-sorted (left two histograms), or Lin-Sca-1-cKit-PU.1GFP+sorted (right two histograms), uninduced (top) or induced with $1 \mu \mathrm{g} / \mathrm{ml} \mathrm{DOX} \mathrm{(bottom)} \mathrm{for} 72 \mathrm{~h}$. Error bars represent standard deviation; $p$ values for all except $F$ were calculated using Student's $t$-test 
studies ${ }^{37}$, maturing erythroid cells are negative for PU.1. To test this, we isolated lin-negative, c-Kit-negative, Sca-1-negative bone marrow cells from Evi1 TO/Rosa26 $6^{r T A} / S p i 1$ ffp $^{\text {T }}$ mice, and separated GFP-negative erythroid progenitors from GFP-positive progenitors. Each population was cultured in vitro and induced with DOX to determine the effects of EVI1 on both PU.1 and Sca-1 expression. Remarkably, Sca-1 induction was confined specifically to the GFP-positive population, indicating that PU.1 is required together with EVIl to induce Sca-1 expression (Fig. 6h). Interestingly, we did not observe induction of GFP expression in either compartment with DOX treatment.

EVI1 binds to $-14 \mathrm{~kb}$ Spi1 upstream regulatory element. Our data indicate a role for both ZF1 and ZF2 of EVI1 in myeloid skewing and in the upregulation of key myeloid regulator, Spi1. To further examine the role of EVI1 in transcriptional regulation of Spi1, we performed Chromatin Immunoprecipitation Sequencing (ChIP-Seq) on DA.1 cells, an EVI1-overexpressing AML. These data (reported in toto previously ${ }^{38}$ ), identified a binding site at $-15.45 \mathrm{~kb}$ from the Spil transcription start site (Fig. 7a), within a previously described regulatory element termed the $-14 \mathrm{~kb}$ upstream regulatory element $(-14 \mathrm{kbURE})^{39}$. We confirmed this binding using ChIP-PCR (Fig. 7b).

Sca-1 upregulation depends on EVI1 binding to $-14 \mathrm{~kb}$ Spi1 URE. To test the role of the -14kbURE in EVI1-mediated upregulation of Spi1/PU.1 and Sca-1, we combined the EviTTO/ Rosa26rtTA alleles with the Spi1-14UREdel (ref. 40; a deletion that encompasses the $-15.45 \mathrm{~kb}$ EVI1-binding element), with the expectation that in mice lacking the $-14 \mathrm{kbURE}$, EVI1 would not be able to bind to the Spil promoter, which would abrogate EVI1mediated upregulation of PU.1; this would allow us to assess the requirement for PU.1 in EVI1-mediated upregulation of Sca-1. We generated Evi1 TO/Rosa26rtTA/Spi1 ${ }^{14 U R E d e l}$ and Evi1TO/ Rosa26 $6^{r t T A}$ mice, and then treated these (as well as WT and Spi $1^{-14 U R E d e l}$ control mice) with DOX (or not) for $72 \mathrm{~h}$; bone marrow was analyzed for upregulation of EVI1, Spi1/PU.1, and Sca-1. As expected, administration of DOX resulted in upregulation of EVIl in both groups harboring the Evi1 TO and Rosa26 $6^{r t T A}$ alleles; there was no induction in WT or Spi1-14UREdel mice (Fig. 7c). DOX treatment resulted in an $83 \%$ increase in Spi1/PU.1 expression in Evi1 TO/Rosa26rtTA mice, but no change in WT or Spi1-14UREdel mice; however, baseline levels of SpiI/ PU.1 transcripts were lower in the latter (Fig. $7 \mathrm{~d}$; ref. ${ }^{40}$ ). In Evi1 ${ }^{T O} /$ Rosa26 $6^{r T A} /$ Spi1 $^{-14 U R E d e l}$ mice, we did observe a DOXinduced increase in Spil/PU.1 transcripts of $45 \%$ despite the deletion of the -14kbURE; while this is less than the increase seen in $E v i 1^{T O} /$ Rosa2 $6^{r t T A}$ mice, the increase of $45 \%$ was still significant $(p<0.001)$, indicating that EVI1-responsive elements exist outside of the $-14 \mathrm{kbURE}$.

Regarding Sca-1 expression: as expected, DOX treatment of Evi1 ${ }^{T O} /$ Rosa26 $6^{\text {rtTA }}$ mice resulted in a more than threefold increase in Sca-1-expressing lineage-negative bone marrow cells (from 8 to $29 \%$ ), while in Evi1 ${ }^{T O} /$ Rosa26 $6^{r T A} /$ Spi1 $^{-14 U R E d e l}$ mice, induction of EVI1 resulted in no change in Sca-1 expression (both $<5 \%$ Sca-1+; Fig. 7e, Supplementary Figure 3). These data indicate that EVI1-induced upregulation of Sca-1 is dependent on upregulation of Spi1/PU.1 by EVI1 via the -14kbURE.

\section{Discussion}

Overexpression of EVI1 is associated with poor-prognosis AML with a median overall survival of 12 months ${ }^{41}$. Despite much investigation, the mechanisms by which EVIl contributes to leukemia have remained obscure. One key observation is that EVI1 is associated specifically with myeloid malignancies ${ }^{42-44}$.
Our findings here offer an insight into this curious association: EVI1 acts through the activation of PU.1 to promote myeloid expansion and interfere with erythropoiesis and lymphopoiesis. Our data indicate that EVI1 overexpression does not by itself cause immediate malignant transformation of hematopoietic cells, but regulates the functional output of the hematopoietic system. Importantly, our analysis also indicates that EVI1 overexpression does not preclude terminal differentiation of the myeloid cells: the EVI1-overexpressing myeloid cells are wellrepresented in the peripheral blood, and by morphology, these appear normal, consistent with previous findings ${ }^{45}$.

This model mimics human myeloid malignancies bearing rearrangements at $3 \mathrm{q} 26$ for the following reasons: (1) the native, unmutated gene, with all of the alternatively spliced isoforms, is upregulated (Supplementary Figure 1H); (2) the levels of EVI1 expression are similar to activated MECOM alleles (Fig. 1b); (3) because we induce EVI1 in the transplanted setting, only hematopoietic cells overexpress EVI1; (4) the overexpressing cells are mixed in with normal cells, as is the case in incipient human myeloid malignancies. While we have not documented EVI1 overexpression in specific lineages within the hematopoietic system in our mouse model, it is known that DOX induction of target genes harboring the tetracycline operons via the rtTA transactivator occurs in multiple compartments within the marrow, and, in general, gene activation in the marrow with this system is quite strong ${ }^{16-18}$. Thus, it is very likely that in our system, EVI1 overexpression occurs in both the HSC compartment, as well as more mature and differentiated derivatives. In humans with 3q26 rearrangements, a super-enhancer from the $3 \mathrm{q} 21 /$ GATA2 locus relocates to be in proximity to $M E C O M^{46}$, resulting in high expression of EVI1 $1^{5}$; whereas the EVI1 is exclusively active in long-term and short-term $\mathrm{HSCs}^{4}$, the GATA2 gene is active both in HSCs and in more mature cells, including erythroid lineage cells ${ }^{47}$. It is known that $3 \mathrm{q} 26$ translocations in myeloid malignancies occur in early $\mathrm{MPPs}^{48}$, and leukemiaassociated translocations have been found in erythroid cells and lymphoid cells ${ }^{49}$. Thus, it is very likely that in humans, the $3 \mathrm{q} 26$ rearranged chromosome is present and expressed as stem/progenitor cells mature to the MPP stage.

While binding of EVI1 to PU.1 has been noted previously ${ }^{50}$, the relationship between EVI1 and Spi1/PU.1 transcriptional regulation has not been investigated previously. Here, we document binding of EVI1 to a previously characterized regulatory element, the -14kbURE, together with EVI1-induced upregulation of PU.1. Strikingly, knocking down PU.1 abrogated myeloid skewing associated with EVI1 overexpression, revealing that PU.1 is necessary for EVI1-induced myeloid expansion. The mechanism by which EVI1 and PU.1 interfere with erythropoiesis likely includes antagonism of GATA1 function; PU.1 has been shown to have this activity ${ }^{51,52}$, and EVI1 is known to bind to GATA1's DNA motif ${ }^{22}$, so it is possible that EVIl itself, in addition to PU.1, impedes GATA1 function in developing erythroid cells.

The most striking transcriptional response induced by EVI1 is upregulation of Sca-1, which is known to have an important role in the granulocytic response to bacteria via TLR4, the receptor for lipopolysaccharides (LPS) $)^{53,54}$; PU.1 is known to play an important role in this ${ }^{53}$. Our data presented here indicate that upregulation of Sca-1 occurs via PU.1 action (Figs. 6, 7). The key findings of our work-that EVI1 overexpression causes lymphoid and erythroid suppression while expanding the myeloid compartment-bears a striking similarity to what is seen during emergency granulopoiesis ${ }^{55}$. Infection with a number of pathogenic organisms results in a shift away from erythropoiesis and lymphopoiesis and towards myelopoiesis ${ }^{56-60}$. The similarities between the effects of EVI1 on the hematopoietic system and the marrow's response to infection suggest either that EVI1 is acting 
a

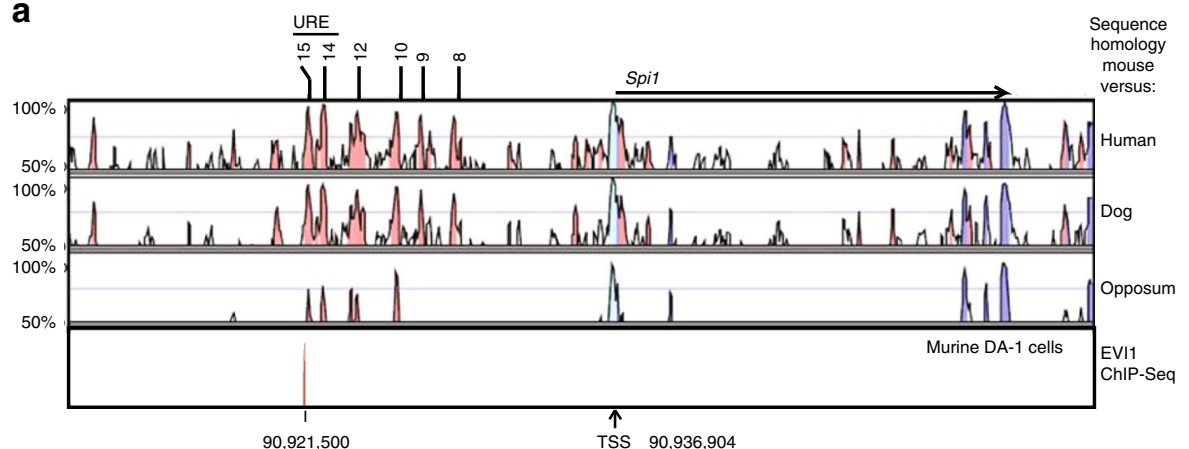

b

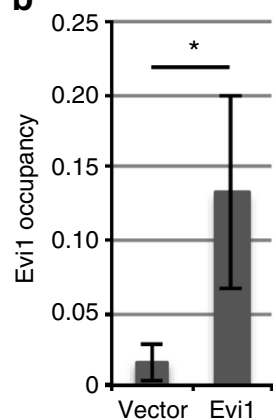

C

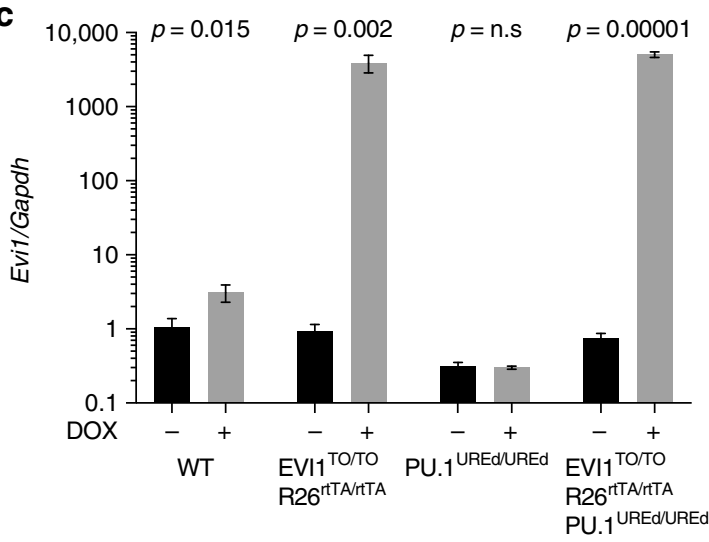

d $2.0 \quad p=$ n.s $\quad p=0.018 \quad p=0.009 \quad p=0.00009$

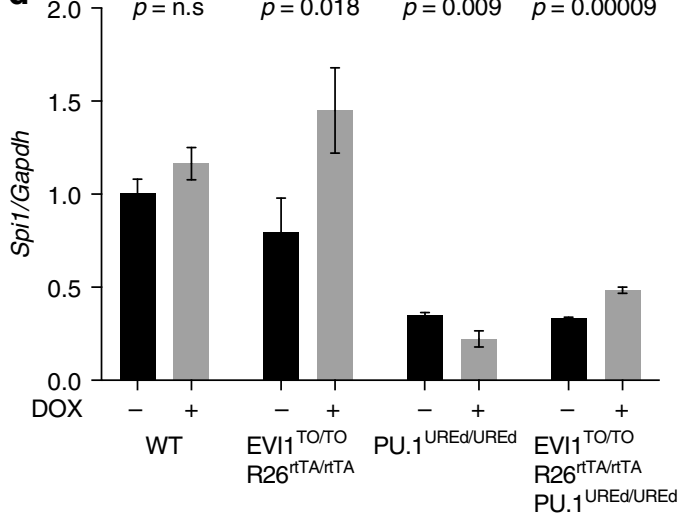

e

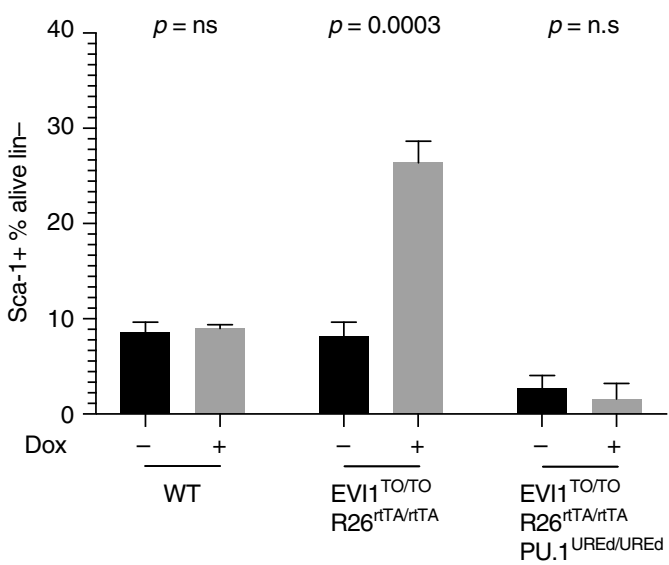

Fig. $7 \mathrm{EVI1}$ binds to the $-14 \mathrm{kbURE}$ of Spi1 and requires this element for Sca-1 upregulation. a Region of mouse genome encompassing the transcription start site (TSS) of Spi1 together with $25 \mathrm{~kb}$ of upstream DNA. Top three bands show peaks of sequence homology between mouse and human, dog, and opossum, as indicated, while the bottom band displays our ChIP-Seq for EVI1 occupancy in the murine myeloid leukemia cell line, DA-1, at -15.45 kb from the Spi1 transcription start site. Also indicated is the location of the -15 to $-14 \mathrm{~kb}$ upstream regulatory element, referred to in the text as $-14 \mathrm{kbURE}$. b ChIP-qPCR data showing EVI1 occupancy at the Spi1-15.45 binding site in EML cells transduced with WT EVI1 or vector control. c, d RT-qPCR for Evi1 (c) or Spi1 (d) on Lin - bone marrow cells, with or without in vivo DOX treatment for $72 \mathrm{~h}$ from four genotypes of mice, harboring either EviT TO/TO Rosa26 rtTA/ rtTA or Spi-1UREd/UREd genotypes, as indicated. e Quantification of Sca-1+cells of WBM from mice with genotypes as indicated either with or without DOX treatment for $72 \mathrm{~h}(n=3)$. For all panels, error bars represent standard deviation; $p$ values calculated with Student's $t$-test

through molecular mimicry, or that it plays a role in the bacterial response. While EVI1 is normally only expressed in the long- and short-term $\mathrm{HSCs}^{4}$, recent findings indicate that it is also induced by LPS (E.A., A.S.P., unpublished findings; ref. ${ }^{61}$ ); and a recently described mutation in EVI1 is susceptible to bacterial infections ${ }^{62}$.

In summary, our findings offer an insight into mechanisms of leukemogenesis, whereby a single nuclear oncoprotein can induce clinically significant suppression of erythropoiesis and lymphopoiesis, while promoting a shift in differentiation that results in a preleukemic expansion of myeloid cells. These findings help to explain the singular association between EVI1 and myeloid malignancies.

\section{Methods}

Mice. The Evi1 ${ }^{T O}$ allele was generated by insertion of the NSTO cassette ${ }^{63}$ via homologous recombination in ES cells using standard techniques ${ }^{64}$. PU.1 ${ }^{\text {gfp }}$ reporter mice ${ }^{37}$ and the Spi-1 UREd mice ${ }^{40}$ have been described. UBC-GFP mice were obtained from Jackson Labs. All procedures involving mice were approved by the institutional animal care and use committees of all participating institutions. 
Harvesting bone marrow. Bone marrow was harvested by one of two methods: (1) flushing the leg long bones the marrow space with flow cytometry (FACS) buffer (PBS, 2\% fetal bovine serum (FBS)) using a $1 \mathrm{ml}$ syringe and 25-gauge needle or (2) grinding femurs, tibia, and pelvis bones $(2 \mathrm{~F} 2 \mathrm{~T} 2 \mathrm{P})$ in FACS buffer using a mortar and pestle ${ }^{65}$.

Flow cytometry and imaging flow cytometry. Marrow harvest, analysis, and transplantations and other procedures were performed as described ${ }^{4,15,65,66}$. Imaging flow was done on ImageStreamX with IDEAS 6.0 (Amnis). Accuri C6, FACSCalibur, LSR II, and FACScan instruments (BD Biosciences) were used for flow cytometric analysis and cell sorting. Supplementary Table 2 has cell-surface marker phenotypes. Cells were stained with antibodies targeted against the appropriate hematopoietic marker for 30 min on ice in the dark using manufacturer's recommended antibody dilutions. Cells were then rinsed once with PBS and resuspended in $1 \times$ PBS, $2 \%$ FBS before analysis. Antibodies used for flow cytometic analysis and cell sorting are listed in Supplementary Table 3. Collection of all flow cytometry data were done using BD FACSDIVA software. Analyses of all flow cytometry data were done using FlowJo version 10 software (TreeStar).

Proliferation and apoptosis. Mice were injected with $2 \mathrm{mg}$ of $\mathrm{BrdU} 60 \mathrm{~min}$ before harvest; incorporation levels were assessed using kit (Cat\#552598; BD Biosciences). Apoptosis rates were determined by flow with anti-annexinV (BD Biosciences).

Cell lines and cell culture. EML cells were provided by Shickwann Tsai and were cultured as described ${ }^{26}$. Methylcellulose colony-forming assays for myeloid progenitors (M, G, and GM-CSF combined) were carried out by plating 10,000 bone marrow cells as previously described ${ }^{67}$. Other cell culture techniques were adapted from previously published protocols ${ }^{4,66}$.

Genetic analysis. RT-qPCR, ChIP, and genomic PCR were performed as described $4,38,66$. The corresponding primers are listed in Supplementary Table 4

Statistical analysis. Data were analyzed using Prism (GraphPad); significance was determined using Student's $t$-test $(\alpha=0.05)$

\section{Data availability}

The authors declare that the data supporting the findings of this study are available within the paper and its supplementary information files. All other relevant data supporting the findings of this study are available from the corresponding author on reasonable request. ChIP-seq data are available in Figshare Repository with the identifier https://doi.org/10.6084/m9.figshare.7043468.

Received: 5 January 2018 Accepted: 21 August 2018

Published online: 12 October 2018

\section{References}

1. Bitter, M., Neilly, M., LeBeau, M., Pearson, M. \& Rowley, J. Rearrangements of chromosome 3 involving bands 3q21 and 3q26 are associated with normal or elevated platelet counts in acute non-lymphocytic leukemia. Blood 66, 1362-1370 (1985).

2. Haase, D. et al. New insights into the prognostic impact of the karyotype in MDS and correlation with subtypes: evidence from a core dataset of 2124 patients. Blood 110, 4385 (2007).

3. $\mathrm{Hu}, \mathrm{Z}$. et al. 3q26.2/EVI1 rearrangement is associated with poor prognosis in classical Philadelphia chromosome-negative myeloproliferative neoplasms. Mod. Pathol. 30, 940-951 (2017).

4. Zhang, Y. et al. PR domain-containing Mds1-Evil is critical for long-term hematopoietic stem cell function. Blood 118, 3853-3861 (2011).

5. Suzukawa, K. et al. Identification of a breakpoint cluster region $3^{\prime}$ of the ribophorin I gene at $3 \mathrm{q} 21$ associated with the transcriptional activation of the EVI1 gene in acute myelogenous leukemias with inv(3)(q21q26). Blood 84, 2681-2688 (1994).

6. Morishita, K. et al. Retroviral activation of a novel gene encoding a zinc finger protein in IL3-dependent myeloid leukemia cell lines. Cell 54, 831-840 (1988).

7. Mucenski, M. L., Gilbert, D. J., Taylor, B. A., Jenkins, N. A. \& Copeland, N. G. Common sites of viral integration in lymphomas arising in AKXD recombinant inbred mouse strains. Oncogene Res. 2, 33-48 (1987).

8. Yatsula, B. et al. Identification of binding sites of EVI1 in mammalian cells J. Biol. Chem. 280, 30712-30722 (2005).

9. Glass, C., Wilson, M., Gonzalez, R., Zhang, Y. \& Perkins, A. S. The role of EVI1 in myeloid malignancies. Blood. Cells Mol. Dis. 53, 67-76 (2014).
10. Louz, D. et al. Erythroid defects and increased retrovirally-induced tumor formation in Evil transgenic mice. Leukemia 14, 1876-1884 (2000).

11. Buonamici, S. et al. EVI1 induces myelodysplastic syndrome in mice. J. Clin. Invest. 114, 713-719 (2004)

12. Yoshimi, A. et al. Evil represses PTEN expression and activates PI3K/AKT/ mTOR via interactions with polycomb proteins. Blood 117, 3617-3628 (2011)

13. Cuenco, G. \& Ren, R. Both AML1 and EVI1 oncogenic components are required for the cooperation of AML1/MDS1/EVI1 with BCR/ABL in the induction of acute myelogenous leukemia in mice. Oncogene 23, 569-579 (2004).

14. Yamazaki, H. et al. A remote GATA2 hematopoietic enhancer drives leukemogenesis in inv(3)(q21;q26) by activating EVI1 expression. Cancer Cell 25, 415-427 (2014)

15. Wilson, M. et al. EVI1 interferes with myeloid maturation via transcriptional repression of Cepba, via binding to two far downstream regulatory elements. J. Biol. Chem. 291, 13591-13607 (2016).

16. Kharas, M. G. et al. Musashi-2 regulates normal hematopoiesis and promotes aggressive myeloid leukemia. Nat. Med. 16, 903-908 (2010).

17. Beard, C., Hochedlinger, K., Plath, K., Wutz, A. \& Jaenisch, R. Efficient method to generate single-copy transgenic mice by site-specific integration in embryonic stem cells. Genesis 44, 23-28 (2006)

18. Giel-Moloney, M., Krause, D. S., Chen, G., Van Etten, R. A. \& Leiter, A. B. Ubiquitous and uniform in vivo fluorescence in ROSA26-EGFP BAC transgenic mice. Genes 45, 83-89 (2007).

19. Hochedlinger, K., Yamada, Y., Beard, C. \& Jaenisch, R. Ectopic expression of Oct- 4 blocks progenitor-cell differentiation and causes dysplasia in epithelial tissues. Cell 121, 465-477 (2005).

20. Barjesteh van Waalwijk van Doorn-Khosrovani, S. et al. High EVI1 expression predicts poor survival in acute myeloid leukemia: a study of 319 de novo AML patients. Blood 101, 837-845 (2003).

21. Cabezas-Wallscheid, N. et al. Identification of regulatory networks in HSCs and their immediate progeny via integrated proteome, transcriptome, and DNA methylome analysis. Cell Stem Cell 4, 507-522 (2014).

22. Perkins, A. S., Fishel, R., Jenkins, N. A. \& Copeland, N. G. Evi-1, a murine zinc finger proto-oncogene, encodes a sequence-specific DNA-binding protein. Mol. Cell Biol. 11, 2665-2674 (1991).

23. Funabiki, T., Kreider, B. L. \& Ihle, J. N. The carboxyl domain of zinc fingers of the Evi-1 myeloid transforming gene binds a consensus sequence GAAGATGAG. Oncogene 9, 1575-1581 (1994).

24. Shimabe, M. et al. Pbx1 is a downstream target of Evi-1 in hematopoietic stem/progenitors and leukemic cells. Oncogene 28, 4364-4374 (2009).

25. Zhang, Y. et al. Targeting a DNA binding motif of the EVI1 protein by a pyrrole-imidazole polyamide. Biochemistry 50, 10431-10441 (2011).

26. Tsai, S., Bartelmez, S., Sitnicka, E. \& Collins, S. Lymphohematopoietic progenitors immortalized by a retroviral vector harboring a dominantnegative retinoic acid receptor can recapitulate lymphoid, myeloid, and erythroid development. Genes Dev. 8, 2831-2841 (1994).

27. Chang, H., Hemberg, M., Barhona, M., Ingber, D. \& Huang, S. Transcriptomewide noise controls lineage choice in mammalian progenitor cells. Nature 453 544 (2008).

28. Pina, C. et al. Inferring rules of lineage commitment in haematopoiesis. Nat. Cell Biol. 14, 287-294 (2012).

29. Iwasaki, H. et al. Distinctive and indispensable roles of PU.1 in maintenance of hematopoietic stem cells and their differentiation. Blood 106, 1590 (2005).

30. Arinobu, Y. et al. Reciprocal activation of GATA-1 and PU.1 marks initial specification of hematopoietic stem cells into myeloerythroid and myelolymphoid lineages. Cell Stem Cell 1, 416-427 (2007).

31. Yamanaka, R. et al. Impaired granulopoiesis, myelodysplasia, and early lethality in CCAAT/enhancer binding protein epsilon-deficient mice. Proc. Natl Acad. Sci. USA 94, 13187-13192 (1997).

32. Kaisho, T. et al. Impairment of natural killer cytotoxic activity and interferon gamma production in CCAAT/enhancer binding protein gamma-deficient mice. J. Exp. Med. 190, 1573-1582 (1999).

33. Parkin, S. E., Baer, M., Copeland, T. D., Schwartz, R. C. \& Johnson, P. F. Regulation of CCAAT/enhancer-binding protein (C/EBP) activator proteins by heterodimerization with C/EBPgamma (Ig/EBP). J. Biol. Chem. 277, 23563-23572 (2002)

34. Sincennes, M.-C. et al. The LMO2 oncogene regulates DNA replication in hematopoietic cells. Proc. Natl Acad. Sci. USA 113, 1393 (2016).

35. Yamada, Y. et al. The T cell leukemia LIM protein Lmo2 is necessary for adult mouse hematopoiesis. Proc. Natl Acad. Sci. USA 95, 3890-3895 (1998).

36. Foudi, A. et al. Distinct, strict requirements for Gfi-1b in adult bone marrow red cell and platelet generation. J. Exp. Med. 211, 909 (2014).

37. Nutt, S. L., Metcalf, D., D’Amico, A., Polli, M. \& Wu, L. Dynamic regulation of PU.1 expression in multipotent hematopoietic progenitors. J. Exp. Med. 201, 221-231 (2005)

38. Glass, C. et al. Global identification of EVI1 target genes in acute myeloid leukemia. PLoS ONE 8, e67134 (2013). 
39. Li, Y. et al. Regulation of the PU.1 gene by distal elements. Blood 98, 2958 (2001).

40. Rosenbauer, F. et al. Acute myeloid leukemia induced by graded reduction of a lineage-specific transcription factor, PU.1. Nat. Genet. 36, 624-630 (2004).

41. Lugthart, S. et al. High EVI1 levels predict adverse outcome in acute myeloid leukemia: prevalence of EVI1 overexpression and chromosome 3q26 abnormalities underestimated. Blood 111, 4329-4337 (2008).

42. Cui, W., Sun, J., Cotta, C. V., Medeiros, L. J. \& Lin, P. Myelodysplastic syndrome with $\operatorname{inv}(3)(\mathrm{q} 21 \mathrm{q} 26.2)$ or $\mathrm{t}(3 ; 3)(\mathrm{q} 21 ; \mathrm{q} 26.2)$ has a high risk for progression to acute myeloid leukemia. Am. J. Clin. Pathol. 136, 282-288 (2011).

43. Secker-Walker, L. M., Mehta, A. \& Bain, B. Abnormalities of 3q21 and 3q26 in myeloid malignancy: a United Kingdom Cancer Cytogenetic Group study. Br. J. Haematol. 91, 490-501 (1995).

44. Wang, W. et al. Clinical and prognostic significance of $3 \mathrm{q} 26.2$ and other chromosome 3 abnormalities in CML in the era of tyrosine kinase inhibitors. Blood 126, 1699-1706 (2015).

45. Metais, J.-Y. \& Dunbar, C. E. The MDS1-EVI1 gene complex as a retrovirus integration site: impact on behavior of hematopoietic cells and implications for gene therapy. Mol. Ther. 16, 439-449 (2008).

46. Groschel, S. et al. A single oncogenic enhancer rearrangement causes concomitant EVI1 and GATA2 deregulation in leukemia. Cell 157, 369-381 (2014).

47. Walsh, J. C. et al. Cooperative and antagonistic interplay between PU.1 and GATA-2 in the specification of myeloid cell fates. Immunity 17, 665-676 (2002).

48. Shi, G., Weh, H.-J., Duhrsen, U., Zeller, W. \& Hossfeld, D. Chromosomal abnormality inv(3)(q21q26) associated with multilineage hematopoietic progenitor cells in hematopoietic malignancies. Cancer Genet. Cytogenet. 96, 58-63 (1997)

49. Crescenzi, B. et al. NUP98/11p15 translocations affect CD34+ cells in myeloid and T lymphoid leukemias. Leuk. Res. 39, 769-772 (2015).

50. Laricchia-Robbio, L., Premanand, K., Rinaldi, C. R. \& Nucifora, G. EVI1 Impairs myelopoiesis by deregulation of PU.1 function. Cancer Res. 69, 1633-1642 (2009).

51. Zhang, P. et al. Negative cross-talk between hematopoietic regulators: GATA proteins repress PU.1. Proc. Natl Acad. Sci. USA 96, 8705-8710 (1999).

52. Zhang, P. et al. PU.1 inhibits GATA-1 function and erythroid differentiation by blocking GATA-1 DNA binding. Blood 96, 2641 (2000).

53. Shi, X. et al. Toll-Like receptor $4 /$ stem cell antigen 1 signaling promotes hematopoietic precursor cell commitment to granulocyte development during the granulopoietic response to Escherichia coli bacteremia. Infect. Immun. 81, 2197-2205 (2013).

54. Melvan, J. N. et al. Alcohol impairs the myeloid proliferative response to bacteremia in mice by inhibiting the stem cell antigen-1/ERK pathway. J. Immunol. 188, 1961-1969 (2012).

55. Manz, M. G. \& Boettcher, S. Emergency granulopoiesis. Nat. Rev. Immunol. 14, 302-314 (2014)

56. Stein, B. The anemia of inflammation. J. Clin. Rheumatol. 18, 437-442 (2012).

57. Zaretsky, A. G., Engiles, J. B. \& Hunter, C. A. Infection-induced changes in hematopoiesis. J. Immunol. 192, 27 (2014).

58. Ueda, Y., Kondo, M. \& Kelsoe, G. Inflammation and the reciprocal production of granulocytes and lymphocytes in bone marrow. J. Exp. Med. 201, 1771 (2005).

59. Nagaoka, H., Gonzalez-Aseguinolaza, G., Tsuji, M. \& Nussenzweig, M. C. Immunization and infection change the number of recombination activating gene (Rag)-expressing B cells in the periphery by altering immature lymphocyte production. J. Exp. Med. 191, 2113 (2000).

60. Liu, A. et al. Hematopoietic stem cell expansion and common lymphoid progenitor depletion requires hematopoietic-derived, cell-autonomous TLR4 in a model of chronic endotoxin. J. immunol. 195, 2524-2528 (2015).

61. Xu, X. et al. EVI1 acts as an inducible negative-feedback regulator of NFkappaB by inhibiting p65 acetylation. J. Immunol. 188, 6371-6380 (2012).
62. Parkinson, N. et al. Mutation at the Evil locus in Junbo mice causes susceptibility to otitis media. PLoS Genet. 3, 1556-1564 (2006).

63. Tanaka, K. et al. Flexible accelerated STOP tetracycline operator-knockin (FAST): a versatile and efficient new gene modulating system. Biol. Psych. 67, 770-773 (2010).

64. Copeland, N., Jenkins, N. \& Court, D. Recombineering: a powerful new tool for mouse functional genomics. Nat. Rev. Genet. 2, 769-779 (2001)

65. Balderman, S. R. et al. Targeting of the bone marrow microenvironment improves outcome in a murine model of myelodysplastic syndrome. Blood 127, 616 (2016)

66. Zhang, Y. et al. Essential role of PR-domain protein MDS1-EVI1 in MLL-AF9 leukemia. Blood 122, 2888-2892 (2013).

67. Palis, J. \& Koniski, A. Analysis of hematopoietic progenitors in the mouse embryo. Methods Mol. Med. 105, 289-302 (2005).

68. Perkins, A. S., Mercer, J. A., Jenkins, N. A. \& Copeland, N. G. Patterns of Evi-1 expression in embryonic and adult tissues suggest that Evi-1 plays an important role in mouse development. Development 111, 479-487 (1991).

\section{Acknowledgements}

This work was funded by National Institutes of Health (NIH) grants: (R01CA175761, and R01CA120313 to ASP), (F31HL143994 to EA); New York State Stem Cell Science awards: (C026423 and C029547 to YZ); funds from the Clinical and Translational Science Institute (CTSA award number UL1 TR002001 from the National Center for Advancing Translational Sciences of the National Institutes of Health) and the Wilmot Cancer Institute at the University of Rochester.We thank Seana C. Catherman, Xiaohui Cui, Stacey Duemel, Ahmed Faisal, Katherine H. Fegan, AnneD. Koniski, Lizz Lamere, Mark Lamere, and Huy Nguyen for their technical assistance.We thank Kenji Tanaka for the pNSTO plasmid and Mineo Kurakawa for the pMys-Evil vector; LeightonGrimes for providinthe SpilGFP mice; Dan Tenen for providing the Spil4UREd mice. We thank ShickwannTsai for providing the EML cell line.

\section{Author contributions}

E.A, M.P.W, K.E.M, A.L, B.J.F. and Y.Z. performed the experiments and created the figures; A.S.P, J.P, and L.M.C. designed the experiments; A.S.P, E.A, K.E.M, M.P.W, and J.P. contributed to the writing of the manuscript.

\section{Additional information}

Supplementary Information accompanies this paper at https://doi.org/10.1038/s41467018-06208-y.

Competing interests: The authors declare no competing interests. The content is solely the responsibility of the authors and does not necessarily represent the official views of the National Institutes of Health.

Publisher's note: Springer Nature remains neutral with regard to jurisdictional claims in published maps and institutional affiliations.

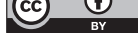

Open Access This article is licensed under a Creative Commons Attribution 4.0 International License, which permits use, sharing, adaptation, distribution and reproduction in any medium or format, as long as you give appropriate credit to the original author(s) and the source, provide a link to the Creative Commons license, and indicate if changes were made. The images or other third party material in this article are included in the article's Creative Commons license, unless indicated otherwise in a credit line to the material. If material is not included in the article's Creative Commons license and your intended use is not permitted by statutory regulation or exceeds the permitted use, you will need to obtain permission directly from the copyright holder. To view a copy of this license, visit http://creativecommons.org/ licenses/by/4.0/.

(C) The Author(s) 2018 